\title{
Design of Conventional and Detonation-Driven Hypervelocity Expansion Tubes
}

\author{
Joel M. Lawson* and Joanna M. Austin ${ }^{\dagger}$ \\ California Institute of Technology, Pasadena, California 91125
}

\begin{abstract}
Freestream disturbances can have a significant impact on studies of boundary layer transition in ground tests, including experiments in high-enthalpy hypersonic facilities such as an expansion tube. Freestream noise reduction in expansion tubes can be achieved by increasing the driver-to-driven pressure ratio $p_{4} / p_{1}$, so we seek methods to increase this ratio in Caltech's HET facility. Towards this end, an in-house modular parametric code has been developed using Python to explore possible modifications to the HET. The effect of increasing the pressure in a passive driver on potential noise reduction is investigated. Optimization of acceleration section length is also explored for conventional drivers.The performance of detonation drivers using $\mathrm{H}_{2}$ and $\mathrm{C}_{2} \mathrm{H}_{2}$ fuels with $\mathrm{He}, \mathrm{Ar}$, and $\mathrm{CO}_{2}$ diluents is examined.
\end{abstract}

\section{Parameters}

\section{Nomenclature}

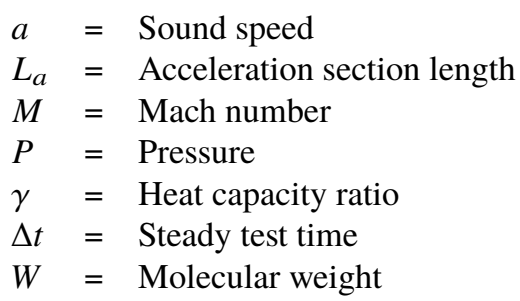

Subscripts

$1=$ Initial driven gas condition

$2=$ Post-shock driven gas condition

$3=$ Post-expansion driver gas condition

$4=$ Initial driver gas condition

$5=$ Initial acceleration gas condition

$6=$ Post-shock acceleration gas condition

$7=$ Freestream test gas condition

$0=$ Total conditions

$s \quad=$ Shock

$p \quad=$ Perfect gas

$r \quad=\quad$ Real gas

opt $=$ Optimal

$H E T=$ With existing HET dimensions

Acronyms

CJ = Chapman-Jouguet

GUI = Graphical User Interface

HET = Hypervelocity Expansion Tube

LFE = Left-facing expansion

RFS = Right-facing shock

SDT $=$ Shock \& Detonation Toolbox

*PhD Candidate, Graduate Aerospace Laboratories, 1200 E. California Blvd. MC 204-45.

$\dagger$ Professor of Aerospace, Graduate Aerospace Laboratories, 1200 E. California Blvd. MC 105-50, Associate Fellow AIAA. 


\section{Introduction}

$\mathrm{P}^{\text {Redicting boundary layer transition is critical to hypersonic vehicle performance and survival under potentially }}$ Pextreme thermal and aerodynamic loads. High-enthalpy, hypersonic conditions which offer the opportunity to examine thermochemical effects can be created in ground tests using impulse facilities: shock tunnels and expansion tubes [1, 2]. Low-temperature hypersonic conditions can be achieved in blowdown tunnels which can also be used to produce a low-disturbance freestream. It is desirable to have a facility that can achieve high-enthalpy, low-disturbance environments for ground testing of transition studies.

In an expansion tube facility, the test gas is initially accelerated by a shock wave and subsequently by an unsteady expansion fan. The first expansion tubes were found to be extremely noisy, with large fluctuations observed in pitot pressure [3]. Early researchers were unsure of the source of this noise. Paull and Stalker demonstrated that the dominant source of noise in the test flow of expansion tubes is the driver gas [4, 5]. Broadband acoustic lateral waves propagate into the driven (test) gas through the primary expansion, becoming focused into a narrow frequency band in the process. If this transmission can be minimized via acoustic impedance, then ultimately the noise that reaches the test flow will be reduced. This can be achieved by minimizing the sound speed ratio $a_{3} / a_{2}$. It can be shown that this ratio is a strong function of the driver-to-driven pressure ratio $P_{4} / P_{1}$. Our primary goal, therefore, is to minimize the sound speed ratio via increase of the driver pressure, or any other means, while preserving other freestream parameters of importance, such as enthalpy, velocity and test time.

This paper is concerned with a calculation toolbox that can be used to evaluate modifications that could be made to the existing HET driver section. There are a large number of variables that affect freestream noise in expansion tubes, so it is beneficial to have a code that can rapidly sweep a large parameter space. Passive, high-pressure driver schemes are considered, together with detonation-driven possibilities.

\section{Development of Code}

\section{A. Numerical vs. Analytical Models}

Expansion tubes have a large amount of tunable parameters: for each of the 3 sections, the gas species, initial pressure and temperature, and sometimes even the length of the section can be altered. If a detonation driver is used, the choice of fuel, oxidizer, diluent and the stoichiometry are additional variables. Therefore, numerical simulation is not a practical option for exploratory work. Instead, a more analytical approach has been taken.

An expansion tube is essentially a two-stage shock tube. The analytical solution to the shock tube is well-known: it assumes a 1-D inviscid wave system where the pressures and velocities must be matched across the contact surface. In an expansion tube, the secondary acceleration of the test gas occurs when the initial shock wave is incident on the driven-accelerator gas interface, initially separated by a diaphragm, generating a transmitted shock and a reflected expansion fan. The interface is modeled by a contact surface, although corrections for diaphragm rupture can be applied [7. The intersection of the right-facing shock polar and the left-facing expansion wave polar in pressure-velocity space is used to calculate the test condition. This can be solved almost instantly for a perfect gas, while a typical desktop computer can find a real-gas solution within several seconds.

To make these calculations tractable, we assume inviscid flow, planar waves, and adiabatic boundaries (walls and contact surfaces). With these, we can model each gas state as a 0-D system connected to each other via wave processes. Across these waves, the chemical composition is either frozen (no reactions or changes from initial fill states) or in equilibrium (infinite reaction rates).

\section{B. Requirements}

Although the present application is to explore noise reduction in the HET specifically, we wanted to develop a code that could be also used for day-to-day current operations of HET. Furthermore, it should be useful for other groups with expansion tubes that may differ from HET.

The following requirements were developed:

- Must be coded in a common, modern language in order to be easily understood and modified by future users.

- Must be portable to most common operating systems.

- Must feature a graphical user interface and error handling for ease of use.

- Must only employ open-source/freeware code and libraries that can be freely distributed.

- Must be modular code so that additional features can be added without substantially altering core routines. 
- Must provide in-built parameter sweeping for rapid design exploration.

- Must output data for deeper analysis in external software.

- Must have external, easily-modified input files for input of gas chemistry data. No data or settings should be hard-coded into the source.

\section{Antecedents}

James et al. developed a parametric analysis code, PITOT, for use with the X2 and X3 expansion tube facilities at The University of Queensland [6, 7]. Their motivation is much the same as described here, and they made several of the same design decisions, notably the use of Python as the coding language. Python was selected due to being free software. The code libraries used for real-gas calculations, i.e. Cantera and Shock \& Detonation Toolbox [8, 9], are natively written in Python. Python also has powerful modules for creating GUIs that are independent of the operating system, e.g. PyQt as chosen here.

The primary difference between our code and PITOT is the user interface. Our code has a full GUI where all input parameters are selected and where results are displayed immediately. At no point does the user need to interact with a command line. Conversely, PITOT runs from a terminal and the user selects the initial state of the facility by writing a configuration file that is read by the code. It is possible to run our code from the terminal by writing custom scripts that bypass the user interface and directly call the underlying solver functions, which may sometimes be desirable for more complicated parameter sweeps.

Due to their differing applications, our code allows for detonation drivers, and wedges in the test flow, whereas PITOT allows for free-piston drivers and nozzles. Given the modular nature of both codes, it would be relatively simple to extend either code to include these features if desired.

PITOT uses NASA's CEA code to for chemistry calculations, whereas we use Cantera, although ultimately the underlying thermodynamic data is from the same source.

\section{Structure}

The code is structured with two basic modes: perfect and real gas. The perfect gas mode is included primarily to speed up the real gas calculations: its solution can be obtained very rapidly and then used as an initial guess for the equivalent real gas problem. It also enables an estimate of the importance of real-gas effects for a given condition.

Back-end functions (thermodynamic models, numerical routines etc.) are grouped into libraries. The GUI layouts are defined in separate file, with a main script that binds the various parts of the GUI to back-end functions. The database of gas thermodynamics and default settings are imported from external files, and the user has the option of outputting results to external files. This modular structure (illustrated in Fig. 11) allows the alteration of any component without breaking the rest of the code.

The expansion tube problem has a large number of variables: each of the 7 gas states has dozens of thermodynamic and kinematic properties to store. Python supports object-oriented syntax, so instead of hundreds of procedural variables, we define classes that represent perfect and real gases so that the code only has to track 7 objects that fully represent each state of the facility. The real gas class inherits all properties and methods of Cantera's thermodynamic and transport models, and extends them to include methods for obtaining sound speed, Reynolds number, Prandtl number etc.

\section{E. Operation}

The GUI is laid out like a virtual version of the facility, with 3 adjacent panels representing the driver, driven, and acceleration sections, and an optional $4^{\text {th }}$ panel representing any test body that might be placed in the flow. The gas species is chosen from a drop-down menu whose entries are defined from an external database that contains perfect-gas thermodynamic data, as well as pointers to the appropriate Cantera input files. The pressure, temperature and length can be altered for each section. The GUI performs real-time checking of these values and will disable calculation if any of the values are outside desired facility operation (e.g. driver pressure less than driven pressure). See Fig.2.

The driver section has additional options. Firstly, it can be specified as 'passive' or 'detonation'. In the former, the user simply chooses the fill pressure (or rather, the diaphragm burst pressure). In the latter, the user first selects from a pre-defined list of combinations of fuel, oxidizer and diluent gases, and the back-end selects an appropriate Cantera file containing all these species and their reactions. A pop-up 'virtual mixer' is used to graphically set the dilution and overall stoichiometry in the pre-detonation mixture. Secondly, instead of specifying the driver pressure, the primary shock speed or Mach number may be fixed instead. In this case, the code solves the shock-tube problem 


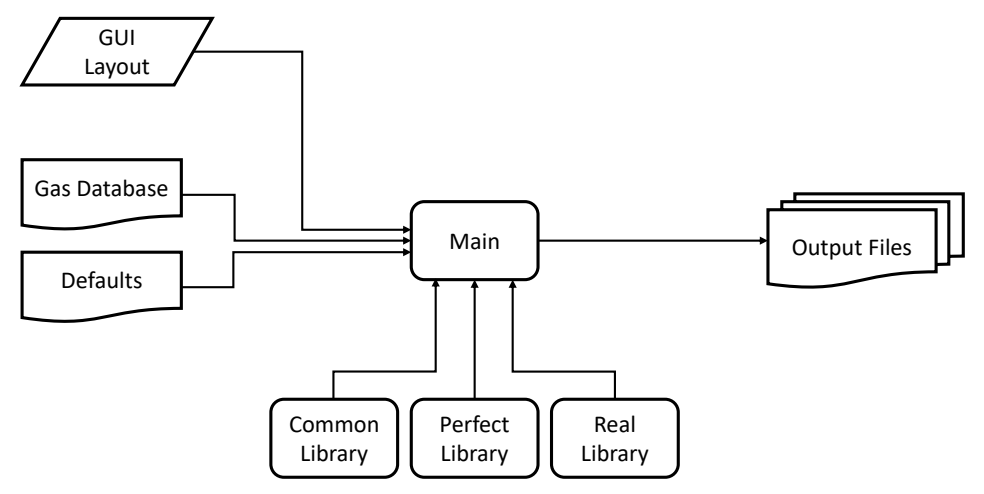

Fig. 1 Schematic of expansion tube code structure.

in reverse and determines the appropriate driver pressure that would result in that shock. This feature is shared with PITOT, where they refer to it as 'experimental mode' since it allows diaphragm burst pressure to be backed out from experimentally-measured shock speeds.

The optional test body panel allows selection of a geometry to be placed in the steady freestream during test time. These calculations are performed subsequently to the full calculation of the facility (i.e. once the test freestream is known). At present, the only available geometry is a wedge. The wedge angle can be chosen, the code outputs whether the shock is attached or detached, and for the former also calculates the oblique shock angle. Due to the modular nature of the code, self-contained functions can be independently developed that calculate flows around different types of geometry as needed. These can then be plugged into the code without requiring external modification and become available as an option in this panel.

The key outputs displayed in the GUI include all the main freestream parameters, as well as statistics for the shock speeds and parameters relevant to the noise-reduction work, such as the sound speed ratio $a_{3} / a_{2}$. More complete data, including all 7 gas states with a full description of their chemical composition etc. can be output to a file. If operating in the real-gas mode, the perfect-gas calculations will be displayed alongside their counterparts with the percentage difference. An $x-t$ diagram is also generated and displayed in the GUI with all the main wave processes illustrated, including reflections of the expansion waves (Fig. 33).

Most usefully for parametric studies, the GUI contains a multiple-calculation mode, where the user selects an independent variable (e.g. driver pressure or acceleration length) and defines a range, as well as constant values for the other facility inputs. The parametric sweep is then plotted on-screen in real-time as the user moves through a list of dependent variables (e.g. freestream enthalpy, test time, primary shock speed). The full dataset for a sweep can also be output to a file for further analysis.

For more complex parametric studies, where multiple independent variables are desired, a library of functions is provided that allows custom scripts to bypass the GUI and directly access the underlying function libraries. Included within this are plotting tools that help address the problem of visualizing the large parameter spaces of expansion tubes. Examples of such studies are given in Section IV 


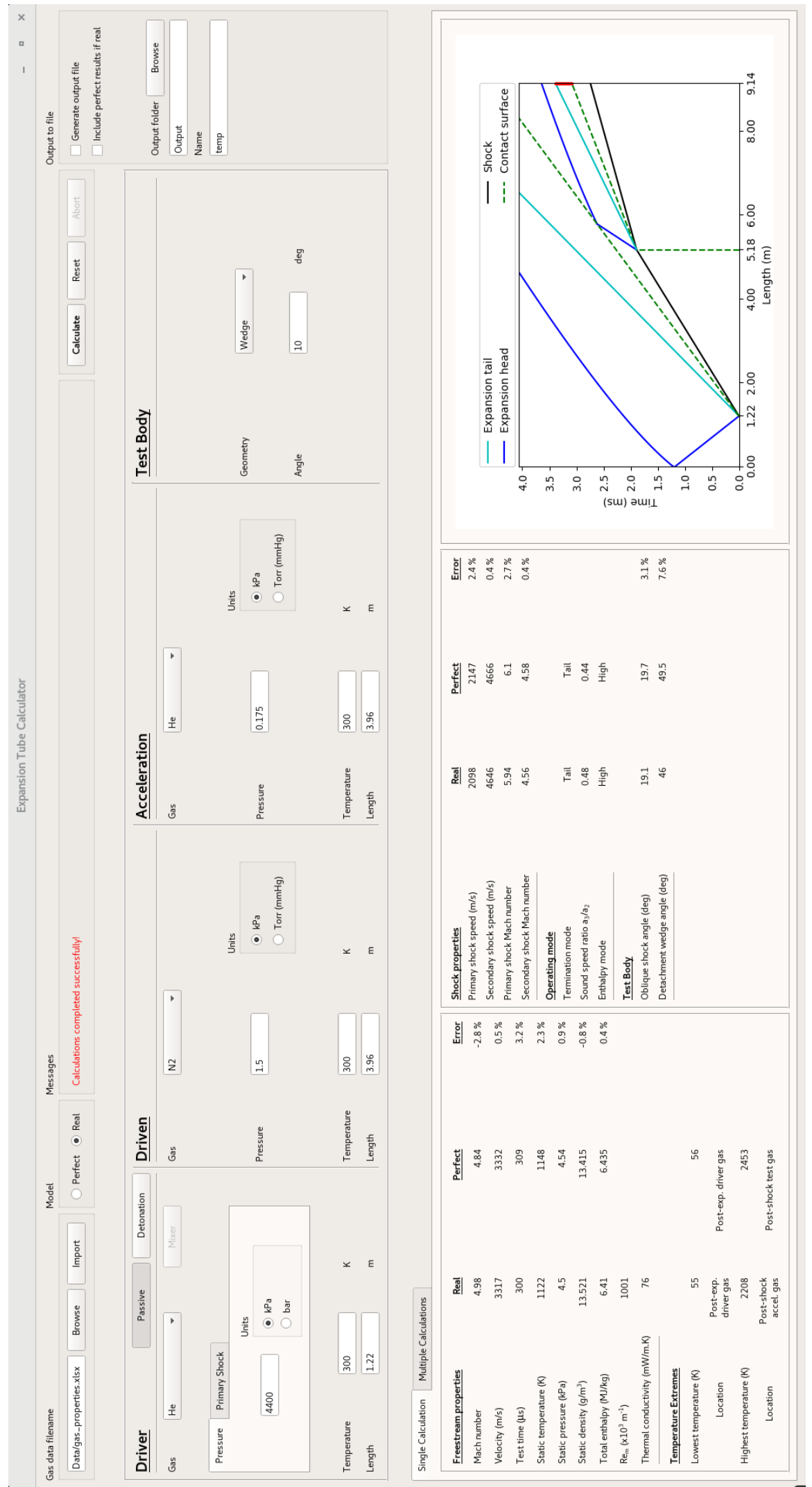

Fig. 2 GUI in single-shot calculation mode. 


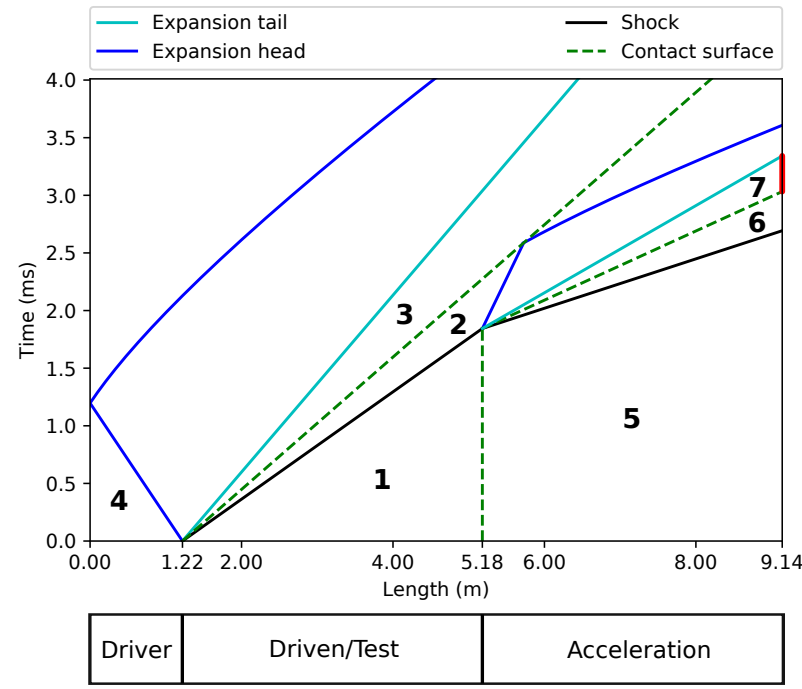

Fig. 3 Automatically-generated $x$ - $t$ diagram for an arbitrary passive-driver condition. Test time indicated in red. Note the curvature of the head characteristics as they reflect through non-simple regions. Annotations give gas state numbers. Section lengths as indicated are for HET $(1.22,3.96$, and $3.96 \mathrm{~m})$.

\section{F. Passive Driver Calculations}

For perfect-gas calculations, we can use analytical expressions for shock jump conditions and isentropic simple unsteady expansions. Combining these yields the well-known shocktube equation (Eq. (1)).

$$
\frac{P_{4}}{P_{1}}=\frac{1+\frac{2 \gamma_{1}}{\gamma_{1}+1}\left(M_{s}^{2}-1\right)}{\left(1-\frac{\gamma_{4}-1}{\gamma_{1}+1} \frac{a_{1}}{a_{4}} \frac{M_{s}^{2}-1}{M_{s}}\right)^{2 \gamma_{4} / \gamma_{4}-1}}
$$

However, this makes the assumption that $u_{1}=u_{4}=0$. While this is true for the 'shocktube' comprising the first stage of an expansion tube, it is not true for the second stage, where the effective driver is state 2 , with $u_{2} \neq 0$. As outlined in Section A, we instead use the $p-u$ polars, Eqs. (2) and (3).

$$
\begin{aligned}
& \text { LFE: } \frac{P_{3}}{P_{4}}=\left(1-\frac{\gamma_{4}-1}{2} \frac{u_{3}-u_{4}}{a_{4}}\right)^{\frac{2 \gamma_{4}}{\gamma_{4}-1}} \\
& \text { RFS: } \frac{P_{2}}{P_{1}}=1+\gamma_{1} \frac{u_{2}-u_{1}}{a_{1}}\left[\frac{\gamma_{1}+1}{4} \frac{u_{2}-u_{1}}{a_{1}}+\sqrt{\left(\frac{\gamma_{1}+1}{4} \frac{u_{2}-u_{1}}{a_{1}}\right)^{2}+1}\right]
\end{aligned}
$$

We want to find the intersection of these polars, i.e. where $P_{2}=P_{3}$ and $u_{2}=u_{3}$. To do this, we use the non-linear root-finding function scipy.optimize. fsolve to find the value of $u=u_{2}=u_{3}$ that minimizes $P_{2}-P_{3}$. Once $u$ is determined, we can fully determine states 2 and 3 by using the shock jump relations (after backing out $M_{s}$ from $\left.P_{2} / P_{1}\right)$ and the Riemann invariant along a $C_{+}$characteristic, respectively. This whole process is then repeated with states 2 and 5 yielding states 6 and 7 .

For real-gas calculations, equivalent analytical expressions for the polars do not exist. SDT contains functions for calculating normal shock jumps with equilibrium or frozen chemistry (e.g. SDToolbox.PostShock_eq). A corresponding function was developed for this code to calculate isentropic unsteady expansions. Like with perfect gases, this still relies on exploiting the Riemann invariant along a $C_{+}$characteristic through the wave. However, here the integral in the expression for the invariant does not simplify down to an algebraic expression. One way of expressing the Riemann invariants is given in Eq. (4). 


$$
\int \frac{\mathrm{d} p}{\rho a} \pm u=\left\{\begin{array}{l}
J^{+} \\
J^{-}
\end{array}\right.
$$

Taking states 3 and 4 as endpoints, using $J^{+}$, and enforcing isentropy, we obtain Eq. [5].

$$
u_{3}=u_{4}-\left.\int_{P_{4}}^{P_{3}} \frac{\mathrm{d} p}{\rho a}\right|_{s}
$$

Cantera-based objects determine their thermodynamic state by setting a pair of variables. In this case, we numerically integrate with respect to $P$, while holding $s$ constant, so at each integration step the object has its pressure altered while always setting the entropy to the known $s_{4}$. We can choose whether to call the equilibrate function at each integration step, thereby modeling either equilibrium or frozen chemistry through the expansion. The function scipy.optimize. quad was chosen to perform the integration as it has features that allow breakpoints to be set in the integration interval to deal with complex functions that change quickly.

To solve the shocktube system, we iterate on the shock Mach number. With $M_{s}$, we use the post-shock functions to obtain a candidate for state 2, including a value for $u_{2}$. Enforcing $P_{2}=P_{3}$, we evaluate Eq. (5) and obtain $u_{3}$. We minimize $u_{2}-u_{3}$ to converge on the actual value of $M_{s}$. This process is accelerated by using $M_{s, p}$ as the initial guess, which is usually within $10 \%$ of $M_{s, r}$.

Once the facility is fully solved, the velocities of the shocks, and expansion wave head and tail characteristics can be found. These are used to construct an $x-t$ diagram, from which test time is determined geometrically. An important feature is the calculation of the reflection of the expansion waves: the primary expansion reflects from the tube end wall, while the secondary expansion reflects from the primary contact surface. Dufrene et al. have previously used a similarity solution to calculate the trajectory of the first reflected characteristic in the non-simple region for the HET. This is particularly important for the secondary expansion as the reflected head may catch up to and overtake the tail. Our code employs this similarity solution, and checks whether test time is terminated by the head or the tail. It also computes the primary reflection, and although further interactions are not calculated, the reflected trajectory is displayed in the $x-t$ diagram to alert the user of niche cases where this reflection may catch up and interfere with downstream wave processes.

At present when calculating conditions with real gases, the code assumes each state reaches chemical equilibrium. However, in some rapid processes, the gas may not have time to equilibrate. From a code perspective, it is a trivial matter to allow for frozen chemistry instead. More challenging is deciding which regime is more appropriate for a given case. A first step would be to add a feature that allows the toggling of chemistry in each process to explore the upper and lower bounds of such effects.

\section{G. Detonation Driver Calculations}

The detonation mode presently being considered is a retonation (upstream-propagating detonation).The driver section is filled with premixed fuel and oxidizer, with ignition occurring near the primary diaphragm [10, 11]. The post-detonation pressure ruptures the diaphragm and the detonation wave propagates to the left, ultimately reaching the driver end wall. In an idealized model, we consider a left-facing Chapman-Jouguet detonation wave, immediately followed by the head of a left-facing expansion wave. It is easy to extend the passive driver code for detonation using this model, because we simply use the post-detonation state $4 \mathrm{a}$ as the effective driver gas. State $4 \mathrm{a}$ can be found from the pre-detonation state 4 using the CJ detonation functions included in SDT.

When dealing with detonation, safety is of paramount concern. A module could be added to the code with additional information about the design and material of the facility, and employ Shepherd's work to estimate the peak dynamic loading on the tube [12]. Related to this, one method of reducing structural loading is to add an extra section upstream of the driver, filled with an inert gas and separated by a weak diaphragm [13]. This section acts as a buffer to help dissipate the detonation wave as it propagates into it. The wave processes in such a section could also be incorporated into the code alongside dynamic loading calculations.

Another safety concern is the composition of the gas remaining in the facility after a shot, which could contain unburnt fuel or toxic combustion products. Since the final composition of all 7 gas states is known, with some extra inputs regarding the dimensions of the tube and dump tank, this feature could be easily added. 


\section{H. Transport Properties}

Viscosity is important to consider in expansion tubes, particularly regarding test time and core flow diameter. At present, the code assumes that the waves move at constant velocities. However, it has been shown both theoretically and experimentally that boundary layer growth in shocktubes causes the shock to decelerate while the contact surface accelerates, eventually reaching a steady separation length [14, 15]. The same phenomenon occurs in expansion tubes [16], and can substantially alter test time from the ideal predictions.

When transport properties are included in the Cantera input files, our code is able to calculate viscosity and thermal conductivity, and consequently Reynolds and Prandtl numbers for any gas state in the facility. These values may be used in future additions to the code to get estimates for boundary layer effects.

\section{Results}

The primary purpose of the results shown in this section is to demonstrate the capabilities of the scripting tools for multi-parameter sweeps. The problem of visualizing the effect of changing multiple input variables was tackled with inspiration from Mollier diagrams: in such a diagram, the axes are enthalpy and entropy, and the plot area shows a mesh of two sets of contours for constant pressure and temperature. In the same way, this code has scripts that allow sweeping over two independent variables, and plotting a contour mesh of these in an output phase space comprised of any two output variables. Two phase spaces commonly chosen represent thermodynamic performance $\left(h_{0,7}\right.$ and $\left.M_{7}\right)$ and flow quality ( $\Delta t$ and $a_{3} / a_{2}$, the latter being a proxy for disturbances in the freestream).

A simple example is given in Fig. 4, where the pressure ratios between adjacent sections of an expansion tube are varied for an expansion tube with section lengths equal to the current HET $(1.22 \mathrm{~m}, 3.96 \mathrm{~m}$, and $3.96 \mathrm{~m}$ for driver, driven, and acceleration sections, respectively). Note that for a perfect gas, only the pressure ratios have an effect, rather than the absolute values of pressure. The annotations give the value of the pressure ratio on each contour.

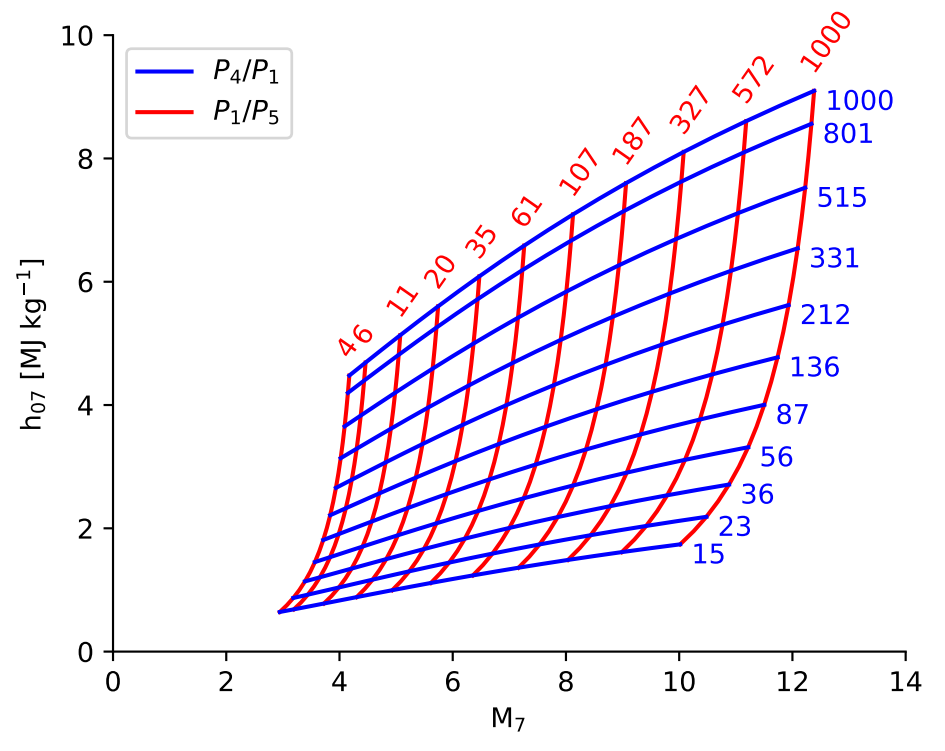

Fig. 4 The effect on freestream Mach number and total enthalpy of changing the two pressure ratios for HET in passive-driver mode (driver: He, driven: $\mathrm{N}_{2}$, acceleration: He). Perfect gas assumed.

Another pair of freestream thermodynamic parameters that are often of interest are $P_{7}$ and $\rho_{7}$. For these, the absolute values of fill pressures do matter, rather than just the ratio as above. We can fix an absolute pressure in one section (usually the test gas) and proceed to sweep over the two ratios that involve this pressure as previously. Using the same conditions as Fig. 4 we obtain Fig. 5

We can also overlay multiple copies of such plots in order to see the effect of changing a gas species, or some assumption in our modeling. For example, Fig. 6 repeats the calculations of Fig. 4 with air as the test gas instead of $\mathrm{N}_{2}$, using both perfect and real gas models. Since the gases are initially at room temperature, we only see minor deviations in the Mach number-total enthalpy phase space, and then only at extreme pressure ratios. The shift is somewhat more 


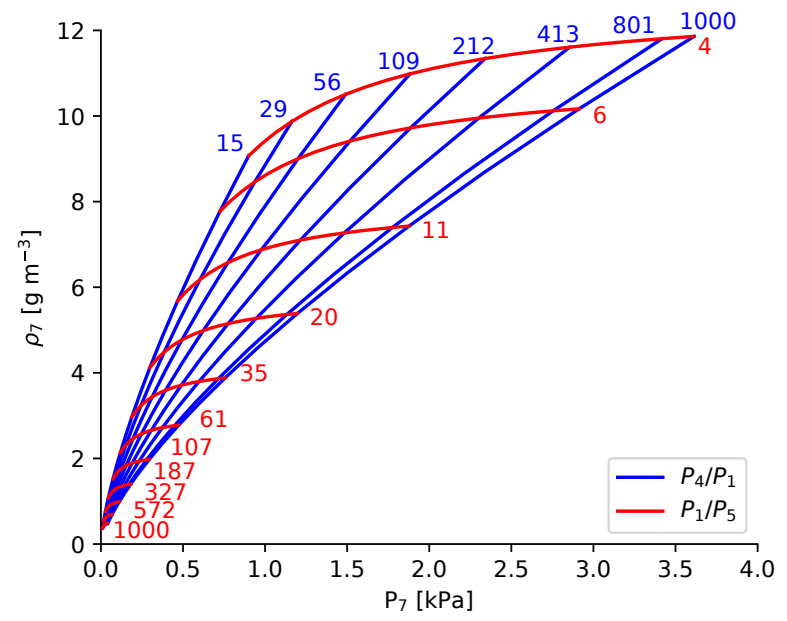

Fig. 5 The effect on freestream static pressure and density of changing the two pressure ratios for HET in passive-driver mode (driver: He, driven: $\mathrm{N}_{2}$, acceleration: $\mathrm{He}$ ). $P_{1}$ fixed at $1 \mathrm{kPa}$. Perfect gas assumed.

noticeable in the sound speed ratio - test time phase space: real gas effects tend to increase the sound speed ratio while decreasing the test time. Since calculating with perfect gas assumptions is equivalent to assuming all processes maintain frozen chemistry, and because the real gas calculations here enforce equilibrium at each state, these plots give bounds on the range of states accessible with different assumptions on reaction rates.

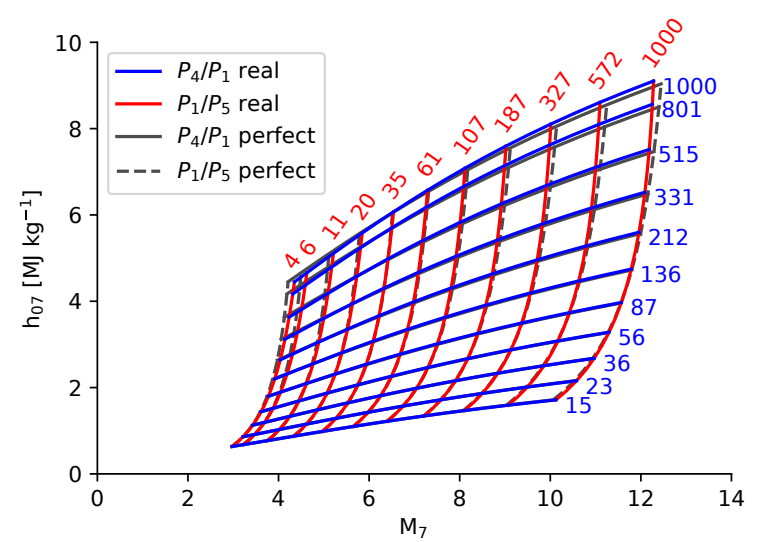

(a) Freestream Mach number - total enthalpy phase space

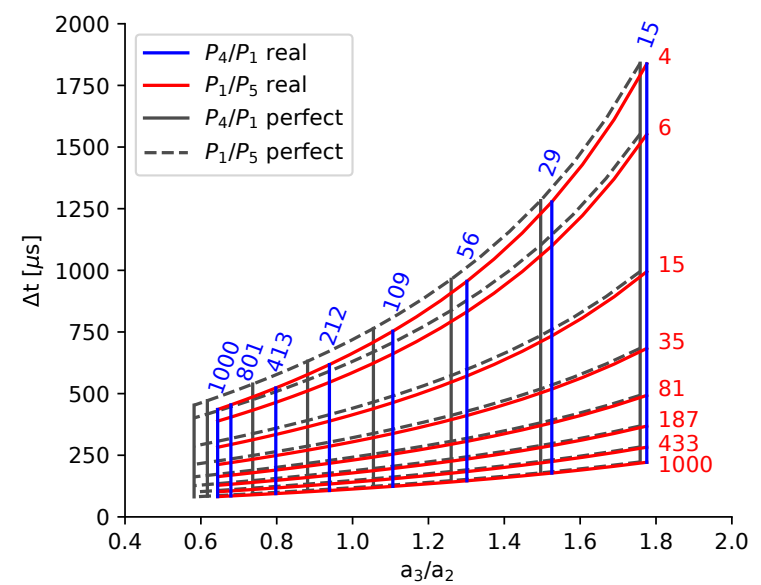

(b) Sound speed ratio - test time phase space

Fig. 6 The effect of changing the two pressure ratios for HET in passive-driver mode (driver: He, driven: air, acceleration: He). Real gas model used with $P_{1}$ fixed at $1 \mathrm{kPa}$. Overlaid on equivalent perfect gas calculation.

For our inviscid assumptions, the driver and driven lengths have no effect on any of the freestream variables, unless the driver length is short enough that the reflected primary expansion is able to interact with downstream wave systems. For conditions where $M_{2}$ is supersonic (which is usual), there is an optimal acceleration length $L_{a \text {,opt }}$ where the secondary expansion tail and the reflected head reach the test section simultaneously, maximizing test time (see Fig. 3). It was found that $L_{a, o p t}$ is not a function of $P_{4}$, but only of $P_{1}$ and $P_{5}$. For given gas species, the data collapses to a single curve if $P_{1} / P_{5}$ and $\Delta t_{o p t} / \Delta t_{H E T}$ or $L_{o p t}$ are used. We observe in Fig. 7 that HET is optimized for $P_{1} / P_{5}$ around 25 , and as this ratio increases, longer acceleration section lengths are required to optimize test time. At very high ratios (required for thermodynamic performance), impractically long sections are indicated, although of course even a smaller length increase would still improve test time. Such plots would be particularly useful for facilities that have variable 
section lengths. Interestingly, we see that $\mathrm{He} / \mathrm{N}_{2} / \mathrm{N}_{2}$ and $\mathrm{He} / \mathrm{CO}_{2} / \mathrm{Ar}$ are almost identical in this phase space, suggesting that further data collapse might be achieved via normalization by some function of $\gamma$ and/or $W$.

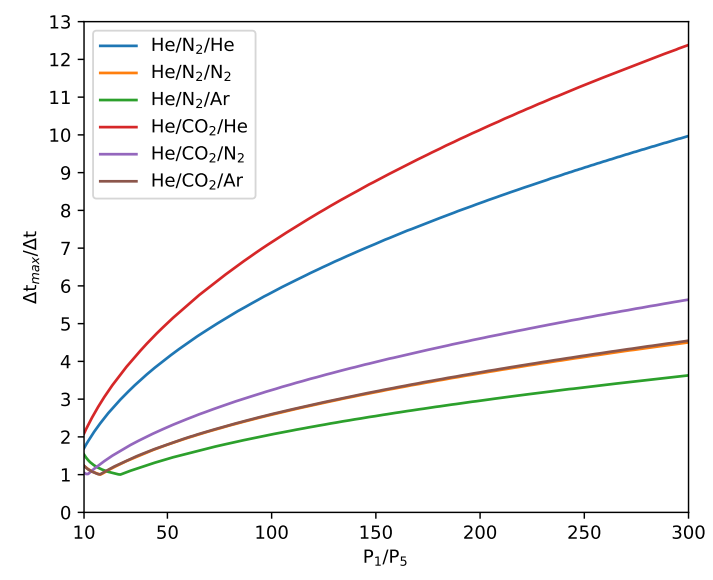

(a) Maximum achievable test time as a multiple of actual test time for current HET dimensions.

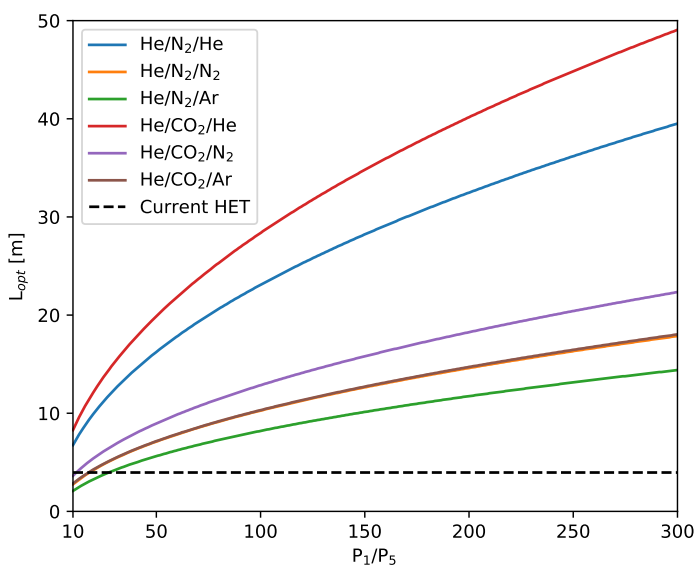

(b) The corresponding acceleration section lengths required to achieve these maximum test times. The current length of HET's acceleration section is indicated.

Fig. 7 Optimization of test time by adjusting acceleration section lengths, for several fill species combinations (passive-driver mode, perfect gas calculations). Note that these are inviscid calculations.

Another set of examples use detonation drivers, and vary the fuel and diluent species, the diluent ratio, and the pre-detonation pressure. The first example uses a stoichiometric $\mathrm{H}_{2}-\mathrm{O}_{2}$ mixture, diluted with various mole fractions of He. The pre-detonation pressure is expressed as a ratio with the driven section pressure, and ranges from 1 to 100. Section lengths are set to the current HET dimensions, and the driven and acceleration sections are kept at a constant pressure ratio of 100, with $\mathrm{N}_{2}$ and $\mathrm{He}$ gases respectively. The results are displayed in Fig. 8 Because post-detonation pressure scales with pre-detonation pressure, we see the same trend of decreasing $a_{3} / a_{2}$ and $\Delta t$ as seen in Fig. 6b In this case, increasing the diluent ratio increases $a_{3} / a_{2}$ because $\mathrm{He}$ is lighter than the combustion product $\mathrm{H}_{2} \mathrm{O}$, thus enhancing $a_{3}$.

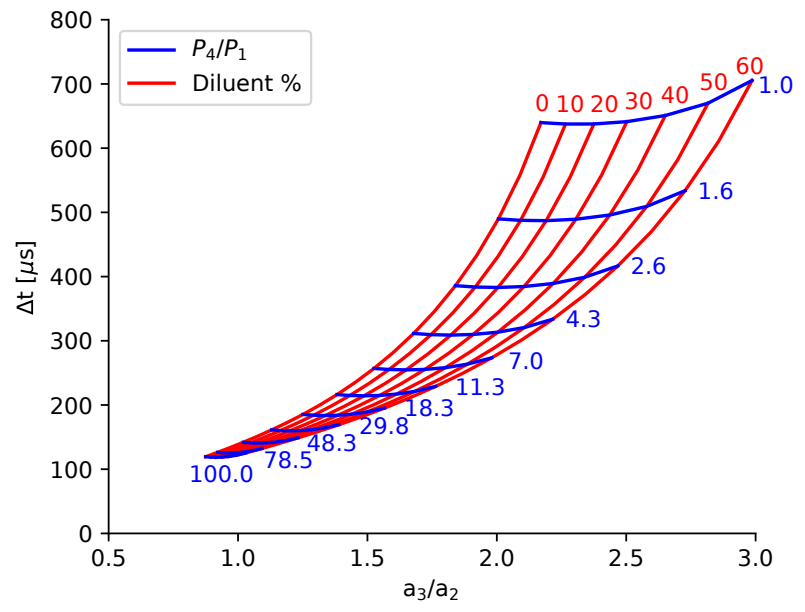

Fig. 8 An example of the effect on sound speed ratio and test time of varying diluent fraction and pre-detonation pressure in a detonation-driven expansion tube. 
A similar example involves varying the diluent species, and holding the diluent fraction fixed at $50 \%$. This was performed for both stoichiometric $\mathrm{H}_{2}-\mathrm{O}_{2}$ and $\mathrm{C}_{2} \mathrm{H}_{2}-\mathrm{O}_{2}$ mixtures, with $\mathrm{He}, \mathrm{Ar}$, and $\mathrm{CO}_{2}$ diluents. These simulations are displayed in Fig. 9 We observe the same trend as previously: increasing diluent $W$ leads to a decrease in $a_{3} / a_{2}$.

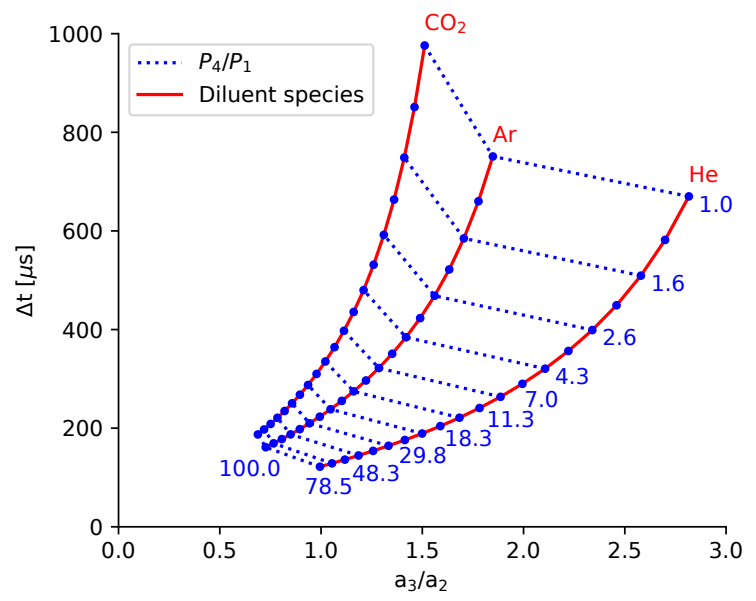

(a) $\mathrm{H}_{2}-\mathrm{O}_{2}$

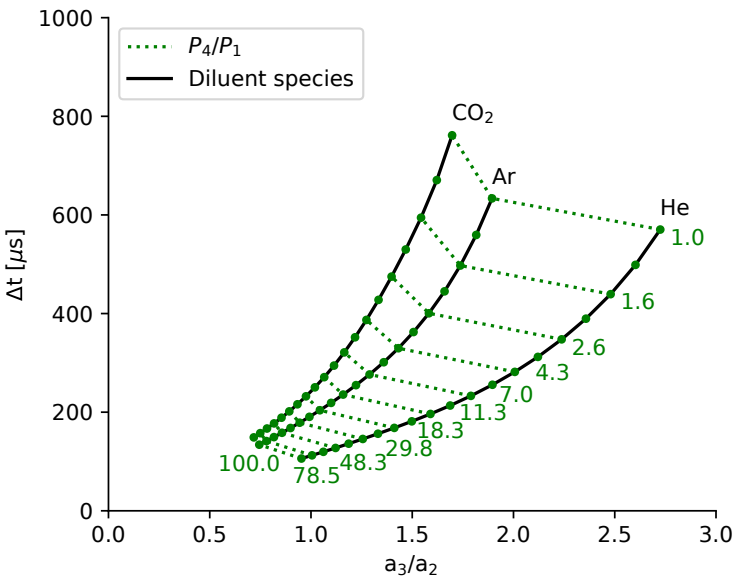

(b) $\mathrm{C}_{2} \mathrm{H}_{2}-\mathrm{O}_{2}$

Fig. 9 An example of the effect on sound speed ratio and test time of varying diluent species and pre-detonation pressure in a detonation-driven expansion tube, for two different fuel species. Dashed lines indicate points with the same pressure ratio. Only every second value labeled for clarity.

\section{Conclusions}

The main purpose of this paper is to detail the development of the rapid simulation code and demonstrate some of its capabilities for both passive and detonation drivers; hence the results in the previous section are mostly illustrative, rather than being a rigorous design study for a new or modified expansion tube facility. Nevertheless, the above cases still offer insight into several design considerations and directions for further investigation.

Firstly, from Fig. 4 we see that all the $P_{1} / P_{5}$ contours become increasingly vertical as $P_{4} / P_{1}$ increases. Hence increases in driver-driven pressure pressure will predominately increase freestream enthalpy without significantly increasing freestream Mach number. For example, when $P_{1} / P_{5}=1000$, changing $P_{4} / P_{1}$ from 15 to 1000 (a $67 \times$ increase) causes $h_{07}$ to increase by $623 \%$ whereas $M_{7}$ only increases by $24 \%$. Conversely, the $P_{4} / P_{1}$ contours become increasingly horizontal as $P_{1} / P_{5}$ decreases, although the effect is not as strong and so the driven-acceleration pressure ratio still significantly influences both parameters.

Because we often desire particular absolute pressures and densities in the freestream, a plot in this phase space can be compared with one that plots $P_{7}$ against $\rho_{7}$ in order to get bounds on absolute (rather than just relative) pressure requirements in each section.

Figs. 6a and 6b illustrate the theory of Paull and Stalker, where increasing the driver pressure ratio decreases the sound speed ratio (desirable) and increases thermodynamic performance (also desirable); however, the test time is reduced for fixed facility section lengths. As expected, the secondary pressure ratio has no effect on the sound speed ratio across the primary contact surface (it cannot, as this ratio is only influenced by the primary wave system), but increasing it also reduces test time. Hence, maximizing freestream enthalpy and Mach number also minimizes test time.

It is difficult to find a set of conditions that allows for arbitrary choice of thermodynamic performance while minimizing sound speed ratio and maximizing test time. However, if we allow variation of section lengths we can try to improve test time while not affecting the other parameters.

\section{Acknowledgments}

The authors would like to thank the Office of Naval Research for supporting this work under the award N00014-16-1-2503, entitled 'Advancing Transition Experiments in High Enthalpy Flows'. 


\section{References}

[1] Dufrene, A., Sharma, M., and Austin, J. M., "Design and Characterization of a Hypervelocity Expansion Tube Facility," Journal of Propulsion and Power, Vol. 23, No. 6, 2007, pp. 1185-1193. doi:10.2514/1.30349, URL http://arc.aiaa.org/ doi/10.2514/1.30349.

[2] McGilvray, M., Doherty, L. J., Morgan, R. G., Gildfind, D., Jacobs, P., and Ireland, P., “T6: The Oxford University Stalker Tunnel," 20th AIAA International Space Planes and Hypersonic Systems and Technologies Conference, International Space Planes and Hypersonic Systems and Technologies Conferences, American Institute of Aeronautics and Astronautics, 2015. doi:10.2514/6.2015-3545, URL/https://doi.org/10.2514/6.2015-3545

[3] Norfleet, G. D., Lacey Jr, J. J., and Whitfield, J. D., "Results of an Experimental Investigation of the Performance of an Expansion Tube,” Tech. rep., Arnold Engineering Development Center, Arnold AFB TN, 1966.

[4] Paull, A., and Stalker, R. J., "The effect on an acoustic wave as it traverses an unsteady expansion," Physics of Fluids A: Fluid Dynamics, Vol. 3, No. 4, 1991, pp. 717-719. doi:10.1063/1.858079, URL/http: / / scitation.aip.org/content/ aip/journal/pofa/3/4/10.1063/1.858079

[5] Paull, A., and Stalker, R. J., "Test flow disturbances in an expansion tube," Journal of Fluid Mechanics, Vol. 245, 1992, pp. 493-521. doi:10.1017/S0022112092000569, URL http: / / www . journals.cambridge.org/abstract_ S0022112092000569

[6] James, C., Gildfind, D. E., Morgan, R. G., Jacobs, P. A., and Zander, F., "Designing and simulating high enthalpy expansion tube conditions," Proceedings of the 2013 Asia-Pacific International Symposium on Aerospace Technology, Japan Soc. for Aeronautical and Space Sciences, 2013, pp. 1-10. URL https://espace.library.uq.edu.au/view/UQ: $317304 / \mathrm{UQ} 317304 \_\mathrm{OA} \cdot \mathrm{pdf}$

[7] James, C. M., Gildfind, D. E., Lewis, S. W., Morgan, R. G., and Zander, F., "Implementation of a state-to-state analytical framework for the calculation of expansion tube flow properties," Shock Waves, Vol. 28, No. 2, 2018, pp. 349-377. doi: 10.1007/s00193-017-0763-3, URL http: // link.springer.com/10.1007/s00193-017-0763-3.

[8] Goodwin, D. G., Moffat, H. K., and Speth, R. L., "Cantera: An object- oriented software toolkit for chemical kinetics, thermodynamics, and transport processes." Tech. rep., 2017. URL www. cantera.org.

[9] Browne, S., Ziegler, J., and Shepherd, J. E., "Numerical Solution Methods for Shock and Detonation Jump Conditions,” GALCIT Report FM2006.006, Mar. 2015. URL http://shepherd.caltech.edu/EDL/publications/reprints/ galcit_fm2006-009.pdf

[10] Lu, F. K., Wilson, D. R., Bakos, R. J., and Erdos, J. I., "Recent Advances in Detonation Techniques for High-Enthalpy Facilities," AIAA Journal, Vol. 38, No. 9, 2000, pp. 1676-1684. doi:10.2514/2.1153, URL http://arc.aiaa.org/doi/10.2514/ 2.1153

[11] Grönig, H., Olivier, H., and Habermann, M., "Development of a detonation driver for a shock tunnel," The Review of High Pressure Science and Technology, Vol. 7, 1998, pp. 879-884. URL https://www.jstage.jst.go.jp/article/ jshpreview1992/7/0/7_0_879/_article/-char/ja/

[12] Shepherd, J. E., "Pressure Loads and Structural Response of the BNL High-Temperature Detonation Tube," Tech. Rep. A-3991, Department of Nuclear Energy, Brookhaven National Laboratory, Sep. 1992.

[13] Yu, H., "Recent developments with gaseous detonation drivers for a shock tunnel," Shock Waves, Springer, 2005 , pp. 65-73. URL http://link.springer.com/chapter/10.1007/978-3-540-27009-6_7

[14] Mirels, H., “Test Time in Low-Pressure Shock Tubes,” Physics of Fluids, Vol. 6, No. 9, 1963, pp. 1201-1214. doi:10.1063/1. 1706887, URL http://scitation.aip.org/content/aip/journal/pof1/6/9/10.1063/1.1706887

[15] Mirels, H., "Shock tube test time limitation due to turbulent wall boundary layer," AIAA Journal, Vol. 2, No. 1, 1964, pp. 84-93. doi:10.2514/3.2218, URL http://arc.aiaa.org/doi/10.2514/3.2218

[16] Shinn, J. L., and Miller III, C. G., "Experimental Perfect-Gas Study of Expansion-Tube Flow Characteristics," NASA Technical Paper TP-1317, Langley Research Center, Hampton, Virginia, Dec. 1978. 